\title{
Stability of Semidiscrete Approximations for Hyperbolic Initial-Boundary-Value Problems: An Eigenvalue Analysis
}

Robert F. Warming,

Richard M. Beam, Ames Research Center, Moffett Field, California

September 1986

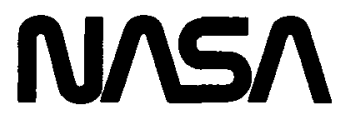

National Aeronautics and

Space Administration

Ames Research Center

Moffett Field, California 94035 


\title{
STABILITY OF SEMIDISCRETE APPROXIMATIONS FOR HYPERBOLIC INITIAL-BOUNDARY-VALUE PROBLEMS: AN EIGENVALUE ANALYSIS ${ }^{1}$
}

\author{
ROBERT F. WARMING AND RICHARD M. BEAM \\ NASA Ames Research Center \\ Moffett Field, CA 94035, USA
}

\section{Introduction}

An evolutionary (time-dependent) partial differential equation (PDE) can be reduced to a system of ordinary differential equations (ODEs) by replacing the spatial derivatives with finite-difference approximations. The resulting approximation is called semidiscrete since the time variable is left continuous. The procedure of reducing a PDE to an ODE system is often called the method of lines since a solution of the ODE system gives an approximation to the PDE solution along $x$ equals constant lines in $(x, t)$ space.

A semidiscrete approximation for a hyperbolic initial-boundary-value problem (IBVP) leads to a complication since, in general, more boundary conditions are required for the semidiscrete approximation than are specified for the PDE. The additional boundary conditions are often called numerical boundary conditions. Any numerical procedure used to provide a numerical boundary condition is called a numerical boundary scheme (NBS).

An essential requirement for a semidiscrete approximation is the convergence (as the spatial mesh is refined) of the approximate solution to the solution of the PDE. If an approximation is consistent, then by the Lax equivalence theorem stability is a necessary and sufficient condition for convergence. It is generally easy to check the consistency of the approximation; however, the stability analysis can be a formidable problem.

Improper treatment of the NBS can lead to instability of the semidiscrete approximation even though one starts with a stable approximation for the pure initial-value problem (IVP) or Cauchy problem. For the purposes of this paper, we will assume that the approximation is consistent and stable (i.e. convergent) for the IVP and will consider only the effect of the NBS on the stability of the semidiscrete IBVP.

For a linear IVP or an IBVP with homogeneous boundary conditions, a semidiscrete approximation (on $J$ spatial mesh intervals) results in a system of ODEs of the form $d u(t) / d t=A \mathbf{u}(t)$ where $u$ is a $J$-component vector, and $A$ is a $J \times J$ matrix. The solution of the homogeneous ODE system can be written as $\mathbf{u}(t)=e^{A t} \mathbf{u}(0)$ where $e^{A t}$ is an exponential matrix. The classical Lax-Richtmyer stability definition is equivalent to the requirement that the matrix norm of $e^{A t}$ be uniformly bounded for $0 \leq t \leq T$ independent of the spatial mesh size. The practical problem is that there is no known simple algebraic test for the uniform boundedness of the matrix norm for hyperbolic IBVPs. Necessary (and sufficient in special cases, e.g., IVPs) conditions can be expressed in terms of the eigenvalues of the matrix $A$; however, for the IBVP the eigenvalue analysis is, in general, intractable and the resulting conditions are not sufficient for stability.

In the 1960s and early 1970s, a stability theory for fully discrete IBVPs was developed by Godunov and Ryabenkii [1], Kreiss [3], Osher [4], and Gustafsson, Kreiss, and Sundström [2]. For the purposes of this paper, we refer to this theory as the GKS theory. Trefethen [6] showed that the main result of the theory has a physical interpretation in terms of group velocity, and Strikwerda [5] extended the theory to semidiscrete approximations.

The GKS theory reduces the stability analysis of a semidiscrete IBVP on a finite domain to the study of three auxiliary problems: the Cauchy problem and the related left- and right-quarterplane problems. The stability of the left- and right-quarter-plane problems is checked by the

\footnotetext{
${ }^{1}$ Preprint for the Proceedings of the Tenth International Conference on Numerical Methods in Fluid Dynamics, Beijing, China, June 23-27, 1986
} 
normal mode analysis. An advantage of the GKS theory is that it provides an algebraic test which is necessary and sufficient for stability but does so at the expense of using a more comnlicated norm (in the stability definition).

Since the Lax-Richtmyer and GKS stability definitions differ, the connection between the eigenvalue analysis for the finite domain problem (which gives necessary Lax-Richtmyer stability conditions) and the eigenvalue or normal mode analysis for the quarter plane problems (which gives necessary and sufficient GKS stability conditions) is rather obscure. In this paper, we consider a direct algebraic comparison between the stability polynomial of the finite domain problem and the polynomials associated with the quarter-plane problems of the GKS theory. We show under what asymptotic conditions the finite-domain analysis leads to a stability polynomial which is consistent with the quarter plane analysis. The asymptotic eigenvalue analysis (finite-domain, $J \rightarrow \infty$ ) establishes the connection between the algebraic tests of the GKS stability theory and the LaxRichtmyer stability definition. In addition, it also leads to a conjecture which gives necessary and sufficient conditions for Lax-Richtmyer stability in terms of the algebraic tests of the GKS theory.

This short paper gives only a brief treatment of our analysis. A detailed exposition (including plots of eigenvalue distributions for various NBSs) is given by the authors in [8]. This paper is restricted to semidiscrete approximations. For fully discrete difference approximations we have recently stated a conjecture [7] which relates the Lax-Richtmyer and GKS stability theories by a generalization of a theorem from linear algebra.

\section{Initial-Boundary-Value Problem for a Model Hyperbolic Equation}

For simplicity we restrict our attention to the stability of semidiscrete approximations to the IBVP for the model hyperbolic equation

$$
\frac{\partial u}{\partial t}=c \frac{\partial u}{\partial x}, \quad 0 \leq x \leq L, \quad t \geq 0
$$

where $c$ is a real constant. Initial data $u(x, 0)=f(x), 0 \leq x \leq L$ are given at $t=0$ and for $c>0$ the problem is well-posed if an analytical boundary condition is prescribed at $x=L$

$$
u(L, t)=g(t) \text { for } c>0 .
$$

\section{A Prototype Semidiscrete Approximation for the Model IBVP}

Let the spatial interval $L$ be divided into $J$ subintervals of length $\Delta x$, i.e., $J \Delta x=L, \quad x=x_{j}=$ $j \Delta x$. On the interior mesh points the spatial derivative $u_{x}$ is replaced by a second order central difference quotient and we obtain the system of ODEs

$$
\frac{d u_{j}}{d t}=\frac{c}{2 \Delta x}\left(u_{j+1}-u_{j-1}\right), \quad j=1,2, \cdots, J-1
$$

where $u_{j}(t)=u_{j}$ denotes the semidiscrete approximation to $u(x, t)$. The right boundary $(x=L)$ is advanced by using the analytical boundary condition (2.2). We assume that the boundary condition is homogeneous, i.e., $g(t)=0$ and for the semidiscrete problem we write

$$
u_{J}=0 .
$$

The spatial computational stencil of (3.1) uses the 3 points $j, j \pm 1$. If we apply (3.1) at the left boundary $(j=0)$, then the stencil protrudes one point to the left of the boundary. It is clear that an additional numerical boundary scheme (NBS) is required to determine the semidiscrete solution. At this boundary $(j=0)$ we change from a centered approximation to a one-sided spatial differencing approximation of $u_{x}$ :

$$
\left.\frac{\partial u}{\partial x}\right|_{0}=\frac{1}{\Delta x}\left[-\alpha u_{2}+(1+2 \alpha) u_{1}-(1+\alpha) u_{0}\right]
$$


where $\alpha$ is a parameter. The approximation (3.3) is first-order accurate for any $\alpha$ except $\alpha=1 / 2$ in which case it is second-order accurate. If we insert (3.3) into the $\operatorname{PDE}(2.1)$ evaluated at $j=0$, there follows the NBS

$$
\frac{d u_{0}}{d t}=\frac{c}{\Delta x}\left[-\alpha u_{2}+(1+2 \alpha) u_{1}-(1+\alpha) u_{0}\right] \text {. }
$$

The system of ODEs (3.1) together with the analytical boundary condition (3.2) and the NBS (3.4) can be written in vector-matrix form as

$$
\frac{d \mathrm{u}(t)}{d t}=A \mathrm{u}(t)
$$

where $\mathrm{u}$ is a $J$-component vector and $A$ is a $J \times J$ matrix.

\section{Lax-Richtmyer Stability of a Semidiscrete Approximation}

The essential element in the stability of a semidiscrete approximation represented by (3.5) is the behavior of the solution as the spatial mesh is refined $(\Delta x \rightarrow 0$, or $J \rightarrow \infty)$. Consequently, one must consider an infinite sequence of ODE systems. The $J$-th member of the sequence is the ODE system (3.5) of dimension $J$. In order to define stability, we need some measure of the magnitude of the solution vector and we use a conventional vector norm $\|\cdot\|$.

Lax-Richtmyer stability for a semidiscrete approximation is defined as follows:

Definition 4.1. A semidiscrete approximation represented by the sequence of ODEs (3.5) is said to be Lax-Richtmyer stable if there exists a constant $K>0$ such that for any initial condition $u(0)$

$$
\|\mathbf{u}(t)\| \leq K\|\mathbf{u}(0)\|
$$

for all $J, J \Delta x=L$ with $L$ fixed and for all $t, 0 \leq t \leq T$ with $T$ fixed.

The eigenvalues $s_{\ell}$ of $A$ are, in general, complex and we write $s_{\ell}=\Re\left(s_{\ell}\right)+i \Im\left(s_{\ell}\right)$. Any semidiscrete approximation for (2.1) will have the factor $c / \Delta x$ on the right-hand side (see e.g. (3.1)). In considering the eigenvalues of the matrix $A$ it is convenient to define a matrix $\hat{A}$ such that $A=(|c| / \Delta x) \hat{A}$. Consequently, the eigenvalues $\hat{s}$ of $\hat{A}$ are related to the eigenvalues $s$ of $A$ by $\hat{s}=s \Delta x /|c|$.

A necessary condition for Lax-Richtmyer stability can be stated as follows:

Lemma 4.1. A necessary condition for a semidiscrete approximation to be Lax-Richtmyer stable is that for all $J, J \Delta x=L$ and all $t, 0<t \leq T$ there exists a nonnegative constant $w$ such that

$$
\max _{\ell} \Re\left(\hat{s}_{\ell}\right) \leq \frac{w}{J}
$$

If the matrix $A$ is a normal matrix, i.e., $A A^{*}=A^{*} A$, then (4.2) is both necessary and sufficient for stability in the $L_{2}$ norm. It is important to realize that a stable semidiscrete approximation can have eigenvalues $\hat{s}_{\ell}$ with positive real parts, but the real parts must approach zero at least as fast as $1 / J$.

\section{Normal Mode Analysis (Quarter-Plane Problems)}

The GKS theory (see introduction) reduces the stability analysis of an IBVP on a finite domain to the study of the three auxiliary problems: the Cauchy problem and the related left- and rightquarter-plane problems. For example, one can obtain the related right-quarter-plane problem from the finite domain semidiscrete problem by fixing $\Delta x$ and the left boundary at $x=0$ and letting $J \rightarrow \infty$. Note that now $L$ is not fixed but $L \rightarrow \infty$ as $J \rightarrow \infty$, and the resulting spatial domain is $[0 \leq x<\infty)$.

The algebraic tests of the GKS theory are carried out by means of the normal mode analysis which is based on the resolvent equations. The resolvent equations for a quarter-plane problem are obtained by substituting

$$
u_{j}(t)=e^{s t} \phi_{j},
$$


where $s$ is a complex constant, into the semidiscrete approximation (i.e., the interior scheme) and the boundary conditions. The resolvent equations consist of a difference equation and bc'undary conditions for the eigenfunction $\phi_{j}$. The general solution of the resolvent equations which is in $L_{2}$ has the form

$$
\phi_{j}=\phi_{0} \kappa^{j}, \quad|\kappa|<1 .
$$

The semidiscrete approximation is GKS stable if there are no nontrivial solutions of the form

$$
\Re(s)>0, \quad|\kappa|<1
$$

and

$$
\Re(s)=0, \quad|\kappa|=1 \quad \text { such that if }\left|\kappa^{*}\right| \rightarrow 1^{-} \text {then } \Re\left(s^{*}\right) \rightarrow 0^{+}
$$

where $\kappa^{*}$ indicates a perturbation off the unit circle and $s^{*}$ a perturbation off the imaginary axis (complex s-plane). We refer to an eigenvalue of the form (5.3) or (5.4) as a GKS eigenvalue or a GKS generalized eigenvalue, respectively.

To illustrate the application of the quarter-plane normal mode analysis we compute the right quarter-plane eigensolutions for the semidiscrete approximation described in section 3 . If we substitute (5.1) into the interior scheme (3.1) and into the NBS (3.4) we obtain

$$
\frac{2 s \Delta x}{c} \phi_{j}=\phi_{j+1}-\phi_{j-1}, \quad \frac{s \Delta x}{c} \phi_{0}=-\alpha \phi_{2}+(1+2 \alpha) \phi_{1}-(1+\alpha) \phi_{0} .
$$

Since (5.5a) is a difference equation for $\phi$, we look for a solution of (5.5) of the form $\phi_{j}=\kappa^{j}$ and obtain

$$
\frac{2 s \Delta x}{c}=\kappa-\frac{1}{\kappa}, \quad \frac{s \Delta x}{c}=(\kappa-1)[-\alpha \kappa+(1+\alpha)] .
$$

By eliminating $s \Delta x / c$ between $(5.6 \mathrm{a}, \mathrm{b})$, one obtains the following cubic equation for $\kappa$ :

$$
q(\kappa)=(\kappa-1)^{2}(2 \alpha \kappa-1)=0 .
$$

The roots of (5.7), i.e., the zeros of $q(\kappa)$, are

$$
\kappa=1,1, \frac{1}{2 \alpha} \quad(\alpha \neq 0) .
$$

We then check to see if there is a GKS eigenvalue or a GKS generalized eigenvalue. We omit the details of the analysis (see [8]) and summarize the GKS stability results as follows:

$$
\begin{array}{ll}
\alpha<-1 / 2 & \text { unstable (GKS eigenvalue) } \\
\alpha=-1 / 2 & \text { unstable (GKS generalized eigenvalue) } \\
\alpha>-1 / 2 & \text { stable (no GKS eigenvalue or generalized eigenvalue). }
\end{array}
$$

\section{Normal Mode Analysis (Finite-Domain Problem)}

The normal mode analysis for the finite domain proceeds in the same way as the right-quarterplane normal mode analysis except that there are two boundaries and, consequently, the analytical boundary condition (3.2) is imposed at $x=L$. The results of the analysis (see [8]) are summarized as follows. An eigenfunction is

$$
\phi_{j}=\kappa^{j}+(-1)^{J+1} \kappa^{2 J}(-1 / \kappa)^{j}
$$

and the corresponding eigenvalue is

$$
2 \hat{s}=\kappa-\frac{1}{\kappa}, \quad \hat{s}=\frac{\Delta x}{|c|} s
$$


where $\kappa$ is a zero of the polynomial $f(\kappa)$ defined by

$$
f(\kappa)=(\kappa-1)^{2}(2 \alpha \kappa-1)+\kappa^{2 J}\left[-(-1)^{J} \kappa^{-1}(\kappa+1)^{2}(\kappa+2 \alpha)\right] .
$$

A complete solution of the eigenvalue problem requires that we find the zeros of the polynomial (6.3). Unfortunately, one cannot obtain the zeros of $f(\kappa)$ analytically and so the eigenvalue problem for the finite domain is intractable. On the other hand, a knowledge of the precise values of the zeros is more information than we need since the algebraic tests for stability require only asymptotic $(J \rightarrow \infty)$ values of the eigenvalues.

\section{Finite Domain vs. Quarter Plane: the Eigenvalue Connection}

The $\kappa$-polynomial (6.3) for the finite domain problem can be written as

$$
f(\kappa)=q(\kappa)+\kappa^{2 J} h(\kappa)
$$

where

$$
q(\kappa)=(\kappa-1)^{2}(2 \alpha \kappa-1), \quad h(\kappa)=\left[-(-1)^{J} \kappa^{-1}(\kappa+1)^{2}(\kappa+2 \alpha)\right] .
$$

We have intentionally split the polynomial $f(\kappa)$ into two parts where the polynomial $q(\kappa)$ is precisely the $\kappa$-polynomial (5.7) associated with the right-quarter-plane problem of the GKS theory.

The notion of stability for the finite domain problem is intimately associated with the solution behavior as the spatial mesh is refined, i.e., $J \rightarrow \infty$. Hence we are primarily interested in the zeros of $f(x)$ for large $J$. In particular, we are interested in the conditions under which the polynomial $f(\kappa)$ reduces to the quarter-plane polynomial $q(\kappa)$ in the limit $J \rightarrow \infty$. The reduction obviously occurs when $|\kappa|^{2 J} \rightarrow 0$ as $J \rightarrow \infty$. The resulting conditions (i.e., the asymptotic behavior of $\kappa$ ) determine the connection between the normal mode analysis of the finite domain problem and the normal mode analysis of the quarter plane problem.

If a zero of the polynomial $f(\kappa)$ can be estimated asymptotically (for large $J$ ), then the corresponding eigenvalue $\hat{s}$ is given by (6.2). One can restate the necessary condition (4.2) for LaxRichtmyer stability by requiring that every eigenvalue $\hat{s}$ of the matrix $\hat{A}$ satisfy

$$
\Re(\hat{s}) \leq \frac{w}{J}
$$

Lax-Richtmyer instability occurs if inequality (6.6) is not satisfied. In the asymptotic analysis to follow, we relate the presence of a GKS eigenvalue or GKS generalized eigenvalue to Lax-Richtmyer instability.

What values of $\kappa$ do we actually need to consider? One can show that there is no loss in generality in assuming that $|\kappa| \leq 1$. In general, the zeros of $f(\kappa)$ which are crucial to the stability (actually instability) of a semidiscrete approximation depend on $J$ and we write $\kappa=\kappa(J)$. Since we are assuming $|\kappa| \leq 1$, we let

$$
|\kappa|=|\kappa(J)|=1-\epsilon, \quad 0 \leq \epsilon(J)<1 .
$$

There are three possible cases to consider:

$$
\begin{array}{lll}
\text { case (1): } & \epsilon(J) \geq \delta>0 & \text { as } J \rightarrow \infty \\
\text { case (2): } & \epsilon(J) \rightarrow 0 & \text { as } J \rightarrow \infty \\
\text { case (3): } & \epsilon(J)=0 & \text { for all } J .
\end{array}
$$

From (6.2) it easy to show that the real part of $\hat{s}$ can be written as

$$
\Re(2 \hat{s})=\Re(\kappa-1 / \kappa)=\frac{a\left(|\kappa|^{2}-1\right)}{|\kappa|^{2}}
$$


where $\kappa=a+i b,|\kappa|^{2}=a^{2}+b^{2}$. In case (1) $\Re(\hat{s}) \neq 0$ as $J \rightarrow \infty$, in case (2) $\Re(\hat{s}) \rightarrow 0$ as $J \rightarrow \infty$, and in case (3) $\Re(\hat{s})=0$ for all $J$.

case (1)

In the first case we assume that $\epsilon$ is strictly bounded away from zero as $J \rightarrow \infty$, i.e. $|\kappa|<1$ as $J \rightarrow \infty$ and consequently

$$
\lim _{J \rightarrow \infty}|\kappa|^{2 J}=0 \text {. }
$$

Then in the limit $J \rightarrow \infty$, the polynomial $f(\kappa)$ reduces to the right-quarter-plane polynomial

$$
q(\kappa)=(\kappa-1)^{2}(2 \alpha \kappa-1) .
$$

We next check to see if the above cubic polynomial has one or more zeros with $|\kappa|<1$. If there is no zero $|\kappa|<1$, then the assumption which led to (6.11) is invalid and we drop consideration of this case. A necessary condition for Lax-Richtmyer stability is that all the eigenvalues must satisfy inequality (6.6) and hence $\Re(\hat{s}) \leq 0$ in the limit $J \rightarrow \infty$. But in obtaining (6.11) we have already taken the limit $J \rightarrow \infty$ and the semidiscrete approximation will clearly be unstable if $\Re(\hat{s})>0$. Consequently, if $q(x)=0$ and (6.2), i.e., the resolvent equations, have a nontrivial solution of the form

$$
\Re(\hat{s})>0, \quad|\kappa|<1,
$$

then the semidiscrete approximation is Lax-Richtmyer unstable. It is clear that (6.12) is identical to (5.3) of the GKS theory. Consequently, case (1) with $\mathfrak{R}(\hat{s})>0$ corresponds to a GKS eigensolution.

case (2)

In case (1), $|\kappa|$ was assumed to be strictly less than unity as $J \rightarrow \infty$. The second case of interest is $|\kappa|<1$ for any finite $J$ but $|\kappa| \rightarrow 1^{-}$as $J \rightarrow \infty$. From (6.7) and (6.8b) we have

$$
|\kappa|=1-\epsilon(J), \quad \epsilon(J) \rightarrow 0 \text { as } J \rightarrow \infty,
$$

and consequently

$$
|\kappa|^{2 J}=[1-\epsilon(J)]^{2 J}=e^{2 J \ln [1-\epsilon(J)]} \approx e^{-2 J \epsilon(J)}, \quad J \rightarrow \infty .
$$

Considering the product $J \cdot \epsilon(J)$ in the limit $J \rightarrow \infty$ there are only two possibilities: either

$$
J \cdot \epsilon(J) \leq K, \quad J \rightarrow \infty, \quad \text { and } \quad \lim _{J \rightarrow \infty}|\kappa|^{2 J}=\text { constant }>0
$$

where $K$ is a positive constant independent of $J$, or

$$
J \cdot \epsilon(J) \rightarrow \infty, \quad J \rightarrow \infty, \quad \text { and } \quad \lim _{J \rightarrow \infty}|\kappa|^{2 J}=0
$$

In general, $\Re(\hat{s})$ is a function of $\kappa$, e.g., (6.9), and using (6.13) we obtain

$$
\Re(\hat{s}) \approx W \epsilon, \quad J \rightarrow \infty
$$

where $W$ is a constant.

First we consider the possibility given by (6.15a). The $\kappa$-polynomial $f(\kappa)$ does not reduce to the quarter-plane polynomial $q(\kappa)$, however

$$
\Re(\hat{s}) \leq \frac{|W| K}{J}
$$

which satisfies the necessary condition (6.6) for Lax-Richtmyer stability. 
For the second possibility given by $(6.15 b)$ the $\kappa$-polynomial $f(\kappa)$ does, in fact, reduce to the quarter-plane polynomial $q(\kappa)$. But from (6.15b) and (6.16) we have for any finite value of $K$, and $J$ sufficiently large,

$$
\Re(\hat{s})>\frac{W K}{J} \quad \text { if } \quad W>0
$$

which violates the necessary Lax-Richtmyer stability condition (6.6).

In summary, if the reduced $\kappa$ polynomial $q(\kappa)$ has a zero $|\kappa|=1$ and a perturbation of the $\kappa$ inside the unit circle $(|\kappa|=1-\epsilon, \epsilon>0)$ leads to a positive $\Re(\hat{s})(W>0$ in (6.16)) the scheme is Lax-Richtmyer unstable. This algebraic test sequence is precisely the GKS test for a generalized eigenvalue (see (5.4))!

case (3)

This final case has the distinct feature that $|\kappa|=1$ is a solution of the $\kappa$ polynomial for all $J$ and consequently $\hat{s}=0$ for all $J$. The $\kappa$ polynomial does not reduce to the quarter-plane polynomial. The GKS analysis does not distinguish this case from case (2) and both are considered in the GKS generalized eigenvalue test. From the point of view of an eigenvalue analysis, the two cases must be treated separately since any instability in the present case derives not from an eigenvalue with a positive real part but from the algebraic growth (as $J \rightarrow \infty$ ) of the norm of the solution due to the normal mode with eigenvalue $\hat{s}=0$. Details of the analysis for this case will be presented elsewhere [8].

\section{Concluding Remarks}

We have presented a brief outline of an analysis that correlates Lax-Richtmyer stability and the GKS normal mode stability analysis. A more complete analysis leads us to conjecture that, except for certain identifiable borderline cases

$$
\text { GKS stability } \Longleftrightarrow \text { Lax-Richtmyer stability, }
$$

i.e., the GKS algebraic tests can be used to check Lax-Richtmyer stability.

\section{References}

[1] S. K. Godunov and V. S. Ryabenkii, "Special stability criteria of boundary value problems for non-selfadjoint difference equations," Russ. Math. Surv. 18, 1-12 (1963).

[2] B. Gustafsson, H.-O. Kreiss and A. Sundström, "Stability theory of difference approximations for mixed initial boundary value problems. II," Mathematics of Computation 26, 649-686 (1972).

[3] H.-O. Kreiss, "Difference approximations for the initial-boundary value problem for hyperbolic differential equations," "Numerical Solutions of Nonlinear Differential Equations, Proceedings Adv. Symposium," Madison, Wis., John Wiley and Sons, New York, 141-166 (1966).

[4] S. Osher, "Stability of difference approximations of dissipative type for mixed initial-boundary value problems. I," Mathematics of Computations $\underline{23}$, 335-340 (1969).

[5] J.C. Strikwerda, "Initial boundary value problems for the method of lines," J. Comput. Physics $\underline{34}$, 94-107 (1980).

[6] L. N. Trefethen, "Group velocity interpretation of the stability theory of Gustafsson, Kreiss, and Sundström," J. Comput. Physics 49, 199-217 (1983).

[7] R. F. Warming and R. M. Beam: "Proceeding of the Workshop on Oscillation Theory, Computation, and Methods of Compensated Compactness," IMA, Minneapolis, Minn., April 1985, Springer-Verlag, New York, (in press).

[8] R. F. Warming and R. M. Beam: "Stability of semidiscrete approximations for hyperbolic initial boundary value problems I: an eigenvalue analysis", NASA TM, 1986. 


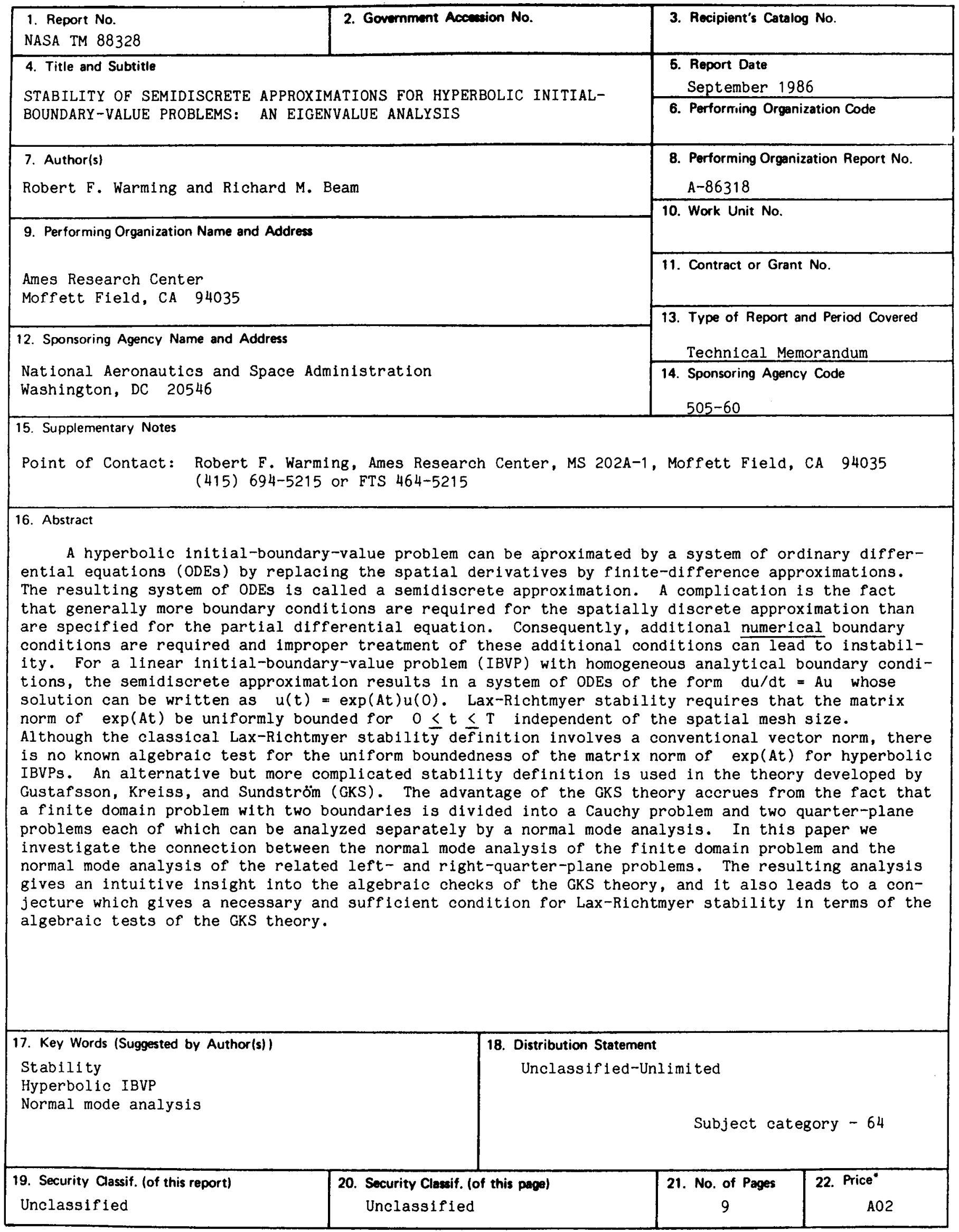

